\title{
On Painter's Paradox: Contextual and Mathematical Approaches to Infinity
}

\author{
Chanakya Wijeratne ${ }^{1,2} \cdot$ Rina Zazkis $^{1}$
}

Published online: 5 May 2015

(C) Springer International Publishing Switzerland 2015

\begin{abstract}
In mathematics education research paradoxes of infinity have been used in the investigation of students' conceptions of infinity. We analyze one such paradox the Painter's Paradox - and examine the struggles of a group of Calculus students in an attempt to resolve it. The Painter's Paradox is based on the fact that Gabriel's horn has infinite surface area and finite volume and the paradox emerges when finite contextual interpretations of area and volume are attributed to the intangible object of Gabriel's horn. Mathematically, this paradox is a result of generalized area and volume concepts using integral calculus, as the Gabriel's horn has a convergent series associated with volume and a divergent series associated with surface area. This study shows that contextual considerations hinder students' ability to resolve the paradox mathematically. We suggest that the conventional approach to introducing area and volume concepts in Calculus presents a didactical obstacle. A possible alternative is considered.
\end{abstract}

Keywords Infinity· Paradoxes · Cognitive conflict · Gabriel's horn

The study of paradoxes of infinity played an important role in investigating students' conception of infinity (e.g. Mamolo and Zazkis (2008), Núñez (1994)). We extend this research by attending to a particular paradox, the Painter's Paradox, which was not yet examined in mathematics education research.

We start by introducing a peculiar object, Gabriel's horn. The dimensions of this object, which are in the heart of the above mentioned paradox, were first studied by Torricelli. To situate the paradox historically, we provide a brief overview of the development of the notion of infinity in mathematics and the debate around

Chanakya Wijeratne

cwijerat@sfu.ca

Rina Zazkis

zazkis@sfu.ca

1 Faculty of Education, Simon Fraser University, 8888 University Drive, Burnaby, BC V5A 1S6, Canada

2 Department of Mathematics, University of Colombo, Colombo 03, Sri Lanka 
Torricelli's discovery. To situate the study of the paradox in mathematics education literature, we provide a brief overview of paradoxes in mathematics and in mathematics education research. We then introduce the Painter's Paradox and follow up with the details of our study, in which a group of Calculus students were invited to consider the Painter's Paradox and address the perceived discrepancy.

\section{Gabriel and His Horn}

Gabriel was an archangel, as the Bible tells us, who "used a horn to announce news that was sometimes heartening (e.g., the birth of Christ in Luke 1) and sometimes fatalistic (e.g., Armageddon in Revelation 8-11)" (Fleron 1999, p.1). The surface of revolution formed by rotating the curve $y=\frac{1}{x}$ for $x \geq 1$ about the $x$-axis is known as the Gabriel's horn (Stewart 2011). This surface and the resulting solid were discovered and studied by Evangelista Torricelli (1608-1647) in 1641. It is unclear why this particular surface came to be known as Gabriel's horn. However, if we attend to what the Bible says about Gabriel and his horn, then we have to ask whether the discovery of this surface by Torricelli was good news or bad news for mathematics. The answer depends on the time period in which this question is asked and the views of infinity in that time period.

At the time of the discovery of Gabriel's horn in the 17th century the term infinity referred to unending processes. The Greek mathematicians of antiquity, up to the time of Aristotle, used the term apeiron to refer to such processes as [endless] counting, successively halving a linear segment (as in Zeno's paradoxes), and evaluating an area by exhaustion. In the 4th century BCE Aristotle explained the idea of infinity as an [endless] process (Kim et al. 2012). He introduced the dichotomy of potential infinity and actual infinity as a means of dealing with paradoxes of the infinite such as Zeno's paradoxes, that he believed could be resolved by refuting the existence of actual infinity. One can think of potential infinity as an endless process, which at every instant of time within a certain time interval is finite. Actual infinity describes a complete entity that encompasses what was potential. Aristotle's potential/actual dichotomy dominated and influenced conceptions of infinity for centuries. Kant (1724-1804), for example, believed that we are finite beings in an infinite world. Therefore we cannot conceive the whole but only the partial and finite. Even more contemporary thinkers such as Poincaré (1854-1912) held largely Aristotelian views (Dubinsky et al. 2005). Then in 1851 Bolzano's work The Paradoxes of Infinity was a serious attempt to introduce infinity into mathematics as an object of study. However, only following Cantor's theory of infinite sets (1874) actual infinity was established as a mathematical object of study (Luis et al. 1991). But Netz and Noel (2011) point out that Archimedes used the notion of actual infinity in his mathematics. But the Greeks made a conscious decision to avoid using actual infinity.

\section{Torricelli's Infinitely Long Solid}

In 1641 Evangelista Torricelli showed that a certain solid of infinite length, now known as the Gabriel's horn, which he called the acute hyperbolic solid, has a finite volume. In 
De solido hyperbolico acuto he defined an acute hyperbolic solid as the solid generated when a hyperbola is rotated around an asymptote and stated the following theorem:

THEOREM: An acute hyperbolic solid, infinitely long [infinite longum], cut by a plane [perpendicular] to the axis, together with a cylinder of the same base, is equal to that right cylinder of which the base is the latus transversum of the hyperbola (that is, the diameter of the hyperbola), and of which the altitude is equal to the radius of the base of this acute body (Mancosu and Vailati 1991, p. 54).

He proved this theorem using curved indivisibles (Fig. 1).

If one rotates a branch of the rectangular hyperbola $y=\frac{1}{x}$ around an asymptote, an infinitely long solid is generated as shown in the above figure. This infinitely long solid is made up of cylindrical indivisibles, the lateral surfaces like PQSR. The cylinder $O A D C$ is made up of circular indivisibles, the cross sections whose radius is $A E$ where $\mathrm{AE}$ is equal to the square root of 2 . The surface $P Q S R$ has area $P Q \times 2 \pi \times O P=\frac{1}{O P}$ $\times 2 \pi \times O P=2 \pi$ and it is equal to the area of the cross section $P N$ of the cylinder $O A D C$. The arbitrarily chosen point $P$ determines an indivisible in the infinitely long solid and an associated indivisible in the cylinder $O A D C$. Since the indivisibles in the two figures, the infinitely long solid and the cylinder $O A D C$, are equal, by the

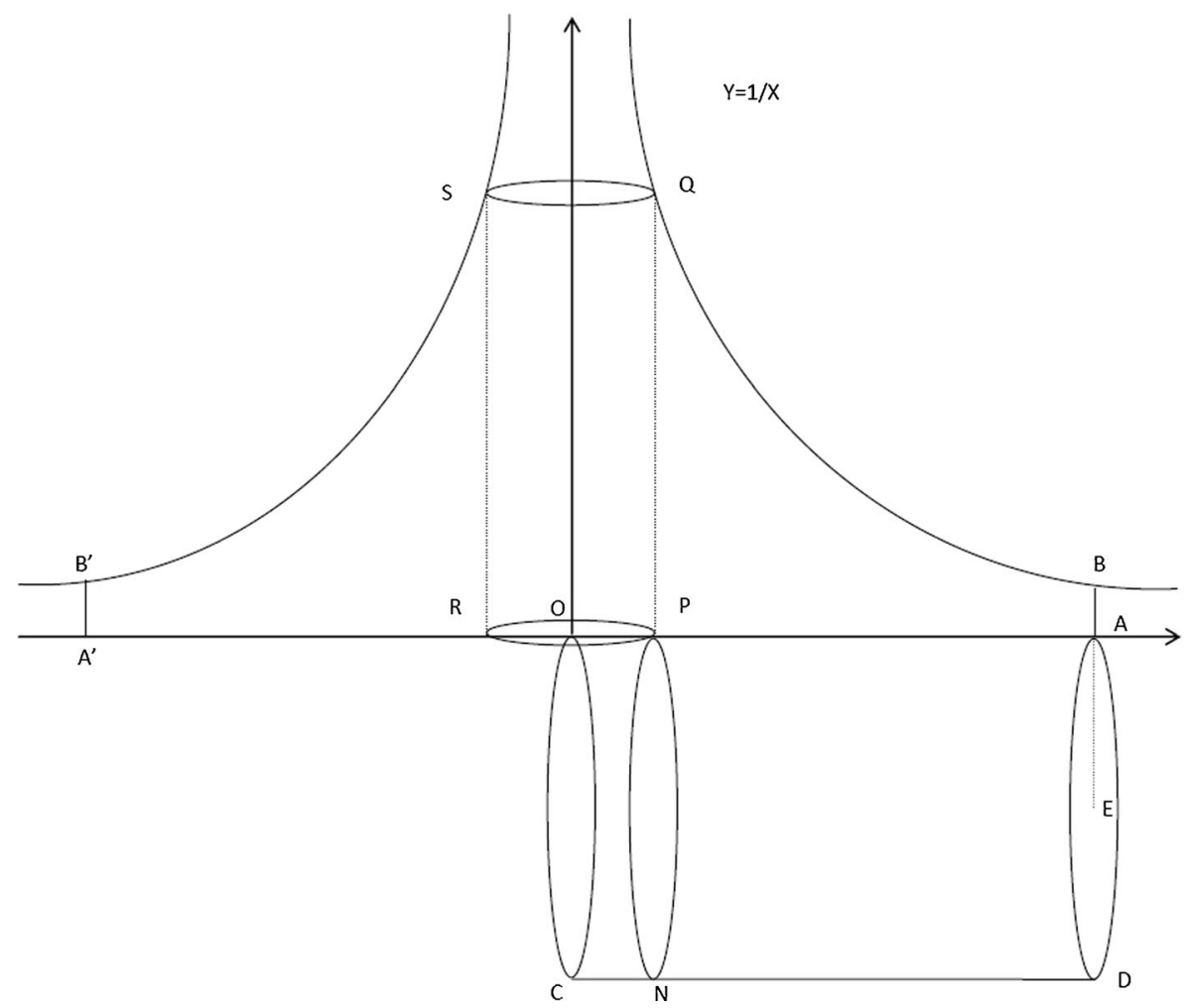

Fig. 1 A cylindrical indivisible of Gabriel's horn at point $P$ and the cylinder Torricelli constructed 
fundamental principle of the theory of indivisibles, the volumes of the two figures are equal. So the volume of this infinitely long solid is $2 \pi \times O A$ (Carroll et al. 2013; Mancosu and Vailati 1991). Mancosu and Vailati (1991) note that Torricelli took for granted the equality of the indivisibles when $P=O$, that is, when the lateral surface degenerates into a straight line.

Mancosu and Vailati (1991) point out that the above theorem brings out the infinitistic nature of Torricelli's result. The notion of actually infinite length is present in the statement of the theorem. The theorem is about the proportion between an infinitely long solid and a finite cylinder. The proof makes sense if the hyperboloid solid is given as infinitely long in actu as the volume of a fixed determinate infinitely long solid with that of a finite cylinder is compared. Also, the volume of the hyperbolic solid is not considered as the limit of a succession of volumes converging to that of the cylinder. "The most striking feature of Torricelli's result, then, is that the acute hyperbolic solid, although finite in volume, is not merely potentially but actually infinite in length" (p. 57).

Torricelli's infinitely long solid gave rise to epistemological and ontological issues at the time of its discovery. It provided non-trivial knowledge about infinity. Also, as Torricelli himself put it "if one proposes to consider a solid, or a plane figure, infinitely extended, everybody immediately thinks that such a figure must be of infinite size" (Ibid., p. 58). Torricelli found his result paradoxical. He was not alone. His contemporaries too found the result paradoxical. It was so paradoxical and counterintuitive that "even 80 years later Bernard de Fontenelle [1657-1757] commented, 'One apparently expected, and should have expected, to find [Torricelli's solid] infinite' in volume” (Ibid., p. 50).

Torricelli's infinitely long solid was a topic in the 17th century debate between the philosopher Thomas Hobbes (1588-1679) and the mathematician John Wallis (16161703 ) on the role of intuition and visual thinking in mathematics. Hobbes insisted that we have ideas only of what we sense and of what we can construct out of ideas we sense. The ideas we sense directly from experience are of finite things. Therefore we cannot construct the idea of an infinite thing. He claimed that anything we conceive must be of finite magnitude, and so when mathematicians say infinite what they usually mean, or ought to mean is indefinite, that is, as great or small as we please. Hobbes believed that the volume of an infinitely long solid must exceed the volume of any finite solid. But, for Wallis, Torricelli's infinitely long solid did not create an issue as long as it was considered as a mathematical object. He shared Leibniz' opinion that it was just as spectacular as, for instance, the fact that the infinite series $\frac{1}{2}+\frac{1}{4}+\frac{1}{8}+\frac{1}{16}+\frac{1}{32}+\ldots$ is equal to 1 (Bråting 2012; Mancosu and Vailati 1991).

Pierre Gassendi (1592 -1655), a philosopher and mathematician, like Hobbes and other empiricists, viewed mathematics as dealing with quantity abstracted from matter and that mathematical concepts are obtained from sensation through a process of abstraction. But he maintained a clear separation between mathematics and physics. Though physicists are constrained by the material nature of the universe, mathematicians, especially geometricians, free themselves through this process of abstraction from the "crassness, obstinancy and impediment of matter" (Gassendi, as quoted in Mancosu and Vailati 1991, p. 61) and create a world in which, in contrast to the real one, there are no indivisible atoms but lines infinitely divisible into other lines, surfaces into other surfaces, and volumes into other volumes. 
And these are the suppositions from which Mathematicians, within the gates of pure and abstract Geometry and almost constituting a kingdom of their own, weave those famous demonstrations, some so extraordinary that they even exceed credibility [fidem], like what the famous Cavalieri and Torricelli showed [ostenderunt] of a certain acute solid infinitely long which nevertheless is equal to a parallelepiped or to a finite cylinder. (Ibid.)

Gassendi concluded that the paradoxical nature of Torricelli's result points to the independence of geometry from physics, as Aristotle had long ago ascertained, and to its structural coherence and beauty. But Mancosu and Vailati (1991) point out that Gassendi's treatment of Torricelli's result is not satisfactory because of his silence about its bold infinitistic nature. They note that Gassendi, like other empiricists in the seventeenth century, maintained that when we say that something is infinite we ought to mean that we are unable to conceive its limits, and therefore one would expect him to make a finitistic reading of Torricelli's result.

Isaac Barrow (1630-1677), a contemporary mathematician of Torricelli, discussed Torricelli's result in a lecture delivered in 1666 on the topic of proportion. This was in connection with the Aristotelian dictum that there is no proportion between the finite and the infinite. He explained that this dictum had been refuted only "in part" and that "there is no proportion between a finite magnitude or quantity and an infinite quantity or magnitude of the same genus" (Barrow, as quoted in Mancosu and Vailati 1991, p. 65). Barrow opined that the reality of geometrical entities is grounded in material existence. This view is compatible with the mathematical existence of Torricelli's solid only if it can actually be instantiated in a piece of matter. Barrow believed that space is infinite but the amount of matter in the universe is finite. This view leads to a possibility of building Torricelli's solid with a finite amount of matter in the infinite space, but it strained Barrow's view of geometrical reality to the point of rupture.

Torricelli's solid raised the issue of the ontological status of geometrical entities and stretched some of the basic intuitive geometrical notions. As he himself pointed out, "in school tracts of geometry one finds figures limited in every side, and among all the solids of which ancient and modern authors have determined the measures with much effort, none, as far as I know, has an infinite extension" (Torricelli, as quoted in Mancosu and Vailati 1991, p. 59). But Mancosu and Vailati (1991) acknowledged that Torricelli's solid is consistent with the definition of a solid given in Euclid; a solid is that which has length, breadth and depth; "but it was apparently at odds with the intuitive universe of geometrical entities constituting the background of the early seventeenth-century geometer" (p. 59).

\section{Paradoxes in Mathematics}

"A paradox has been described as a truth standing on its head to attract attention. Undoubtedly, paradoxes captivate. They also cajole, provoke, amuse, exasperate, and seduce. More importantly, they arouse curiosity, stimulate, and motivate" (Kleiner and Movshovitz-Hadar, 1994, p.1). A paradox, in its use, is a statement that contradicts commonly held notions. 
Paradoxes in mathematics have a long history. Zeno's paradoxes seem to be earliest in the recorded history and they appeared in the 5th century BCE. Zeno of Elea (ca. 490 BCE - ca. 430 BCE) devised arguments, known as Zeno's paradoxes, apparently to defend the doctrine of his teacher Parmenides that there is only one, not many, in the way of truth and that motion and change are impossible. The most famous of Zeno's paradoxes, Achilles and the Tortoise, "says that the slowest moving object cannot be overtaken by the fastest since the pursuer must first arrive at the point from which the pursued started so that necessarily the slower one is always ahead" (Kline 1990, p. 36). Quine (1976) noted that "more than once in history the discovery of a paradox has been the occasion for major reconstruction at the foundations of thought" (p. 3), and this was certainly the case with Zeno's paradoxes. As mentioned earlier, in the 4th century BCE Aristotle introduced the dichotomy of potential infinity and actual infinity as a means of dealing with paradoxes of the infinite, such as Zeno's paradoxes. Another example is Russell's paradox, discovered in 1901, that arises if $R=\{x / x \notin x\}$ is considered as a set as it leads to $R \in R \Leftrightarrow R \notin R$. Russell's paradox shook the foundations of mathematics and created a crisis, and led to axiomatic development of set theory.

Torricelli's infinitely long solid, a paradox according to all accounts of the views of it held in the seventeenth century, brought to light the inadequacy of the empiricist notion of infinity for seventeenth-century mathematical developments. Later in the century John Locke admitted that inadequacy in a long discussion of the empiricist view of infinity (Mancosu and Vailati 1991).

\section{Paradoxes in Mathematics Education Research}

In mathematics education research paradoxes have been used as a lens on student learning. Movshovitz-Hadar and Hadass $(1990,1991)$ investigated the role mathematical paradoxes can play in the pre-service education of high school mathematics teachers. They concluded that

A paradox puts the learner in an intellectually unbearable situation. The impulse to resolve the paradox is a powerful motivator for change of knowledge frameworks. For instance, a student who possesses a procedural understanding may experience a transition to the stage of relational understanding (MovshovitzHadar and Hadass 1991, p. 88).

Núñez (1994) used Zeno's paradox, the Dichotomy, in a progressive manner to investigate how the idea of infinity in the small emerges in the minds of students aged $8,10,12$, and 14 . He concluded that before the age of 12 there is a profound and striking ontological difference between infinity in the large and infinity in the small. For example, though 8 year-olds can conceive of the notion of endless in their consensual world they cannot see the distinction "infinity in the small". Mamolo and Zazkis (2008) used the Hilbert's Grand Hotel paradox and the Ping-Pong Ball Conundrum to explore the naive and emerging conceptions of infinity of two groups of university students with different mathematical backgrounds; undergraduate students in Liberal Arts Programs and graduate students in a Mathematics Education Master's Program. They found that the liberal arts students were more likely to find the idea of a bounded 
infinite set, such as infinitely many time intervals within a finite time interval, problematic. They noted that this difficulty exemplifies the resistance towards actual infinity and it may also be attributed to specific challenges regarding the "infinitely small" in comparison to infinitely large.

\section{Painter's Paradox}

Gethner (2005) says that the paradox of Gabriel's horn is a favourite topic of many Calculus teachers. The paradox of Gabriel's horn, to which he refers, is not Torricelli's infinitely long solid that aroused astonishment and created disbelief among mathematicians and philosophers alike in the seventeenth century. It is the Painter's Paradox given below:

The inner surface of the Gabriel's horn is infinite; therefore an infinite amount of paint is needed to paint the inner surface. But the volume of the horn is finite $(\pi)$, so the inner surface can be painted by pouring a $\pi$ amount of paint into the horn and then emptying it.

In Calculus textbooks Gabriel's horn having infinite surface area and finite volume is not discussed as paradoxical. For example Single variable calculus: Early transcendentals by Stewart (2011) talks about Zeno's paradoxes, but surface area and volume calculations of Gabriel's horn are given as exercises without any comment.

However, in the textbook Calculus - A Complete Course by Adams and Essex (2010), the above Painter's Paradox is mentioned in a slightly different way in the section Volumes by Slicing - Solids of Revolution. An example elaborates on how to "Find the volume of the infinitely long horn that is generated by rotating the region bounded by $y=\frac{1}{x}$ and $y=0$ and lying to the right of $x=1$ about the $x$-axis" (p. 393). After showing that the volume of the horn is $\pi$ cubic units, it is noted:

It is interesting to note that this finite volume arises from rotating a region that itself has an infinite area: $\int_{1}^{\infty} d x / x=\infty$. We have a paradox: it takes an infinite amount of paint to paint the region but only a finite amount to fill the horn obtained by rotating the region. (How can you resolve this paradox?) (p. 393)

While the paradox is mentioned, the resolution is not elaborated upon. Using the disk method the volume of the Gabriel's horn can be calculated by $\int_{1}^{\infty} \pi\left(\frac{1}{x}\right)^{2} d x$ where $\pi\left(\frac{1}{x^{2}}\right)$ is the cross sectional area of the horn perpendicular to the $x$-axis. This improper integral converges to $\pi$ :

$$
\int_{1}^{\infty} \pi\left(\frac{1}{x}\right)^{2} d x=\lim _{a \rightarrow \infty} \int_{1}^{a} \pi\left(\frac{1}{x}\right)^{2} d x=\lim _{a \rightarrow \infty}\left(\pi \int_{1}^{a} \frac{1}{x^{2}} d x\right)=\lim _{a \rightarrow \infty}\left(\pi\left[-\frac{1}{x}\right]_{1}^{a}\right)=\lim _{a \rightarrow \infty}\left(\pi\left[1-\frac{1}{a}\right]\right)=\pi .
$$

The volume of the Gabriel's horn can also be calculated using cylindrical shells too:

$$
\int_{0}^{1}(x-1) 2 \pi y d y=\lim _{a \rightarrow 0^{+}} 2 \pi \int_{a}^{1}(x y-y) d y=2 \pi \lim _{a \rightarrow 0^{+}} \int_{a}^{1}(1-y) d y=\pi .
$$


This is similar to Torricelli's method of finding the volume using indivisibles. The surface area of the horn is given by the improper integral:

$$
\int_{1}^{\infty} 2 \pi\left(\frac{1}{x}\right) \sqrt{1+\left(-\frac{1}{x}\right)^{2} d x} .
$$

Now,

$$
\lim _{a \rightarrow \infty} \int_{1}^{a} 2 \pi\left(\frac{1}{x}\right)\left(\sqrt{1+\left(-\frac{1}{x^{2}}\right)^{2}} d x \geq \lim _{a \rightarrow \infty} \int_{1}^{a} 2 \pi\left(\frac{1}{x}\right) d x=\lim _{a \rightarrow \infty} 2 \pi[\ln x]_{1}^{a}=\lim _{a \rightarrow \infty} 2 \pi[\ln a-\ln 1]=\infty .\right.
$$

Therefore the surface area is infinite.

Is an infinite amount of paint needed to paint the inner surface of the Gabriel's horn? According to Stewart (2011), "we want to define the area of a surface of revolution in such a way that it corresponds to our intuition. If the surface area is $A$, we can imagine that painting the surface would require the same amount of paint as does a flat region with area $A$ " (p. 569). Painting a surface means applying a coat of paint on the surface. So the amount of paint needed refers to the volume of paint. To paint a flat surface of infinite surface area with a uniform thickness $c$ one needs an infinite amount (volume) of paint as the amount needed to paint a finite area $A$ is $c A$ and $\lim _{A \rightarrow \infty} c A=\infty$. So intuitively one can see that an infinite amount (volume) of paint is needed to paint the inner surface of the Gabriel's horn, as the surface area of the Gabriel's horn is infinite.

Painter's Paradox highlights the paradoxical nature of Torricelli's infinitely long solid having finite volume and infinite surface area. Though Calculus textbooks do not discuss Gabriel's horn having infinite surface area and finite volume as paradoxical, some Calculus textbooks acknowledge this by mentioning the Painter's Paradox.

\section{The Concepts of Area and Volume in Calculus}

How are the concepts of area and volume dealt with in Calculus textbooks? In Stewart's Single variable calculus: Early transcendentals (2011), which offers a conventional approach, the topic of area starts with an area problem: finding the area of the region that lies under the graph of $y=f(x)$ from $x=a$ to $x=b$ where $a<b$ and $f$ is a nonnegative real valued continuous function. Without defining explicitly the concept of area, and assuming that this is part of the prior knowledge of the reader, the area of a rectangle is taken as the product of its length and width. Then the area of a region with straight edges can be found using rectangles. But the area of a region with curved sides has to be approximated by rectangles through a limit process. So the area problem is solved by defining the area of the region that lies under the graph of $y=f(x)$ from $x=a$ to $x=b$ where $a<b$ and $f$ is a nonnegative real valued continuous function as the limit of Riemann sums

$$
\lim _{n \rightarrow \infty} \sum_{i=1}^{n} f\left(x_{i}^{*}\right) \Delta x_{i}
$$

where $a=x_{0}, x_{i}, \ldots, x_{n}=b$ is a partition of $[a, b], \Delta x_{i}=x_{i}-x_{i-1}$ and $x_{i}^{*} \in\left[x_{i-1}, x_{i}\right]$ for $i=1,2, \ldots, n$ are sample points for the partition. 
The limit is taken over all possible partitions and sample points. This limit is denoted by the definite integral $\int_{a}^{b} f(x) d x$.

A similar approach is adopted for volume. For example, the textbook authors Adams and Essex (2010) indicate: "we will not attempt to give a definition of volume but will rely on our intuition and experience with solid objects to provide enough insight for us to specify the volumes of certain simple solids" (p. 390). By taking the volume of a rectangular box with length $l$, width $w$, and height $h$ as $l w h$, the volume of a bounded solid is first approximated by Riemann sums and then defined by a definite integral as the limit of Riemann sums. Similarly arc length and area of a surface of revolution are defined by definite integrals.

\section{Generalized Area and Volume}

In Calculus books the definite integral is generalized to include integration over infinite intervals and of unbounded integrands. These integrals are called improper integrals. One or two examples of calculating area under the graph of a function over an infinite interval, and of an unbounded function are done or discussed as motivation to introduce improper integrals. But the emphasis is on improper integrals, not on finding areas or volumes of unbounded regions. Commenting on the volume calculation of the Gabriel's horn in Calculus - a complete course by Adams (2002), Bråting (2012) notes that the textbook does not explain that this kind of volume calculation through an improper integral generalizes the volume concept to an infinite object. He further comments that,

[...] one problem of not mentioning that the volume concept has been generalized could be that students believe that we calculate a volume of an "everyday object". Another problem could perhaps be that students think that it is possible to intuitively based on "everyday objects" understand [sic] that the volume of the above horn [Gabriel's horn] is finite (p. 5).

Even in Adams and Essex (2010), a later edition of the textbook, the generalization of the volume concept, or the area concept, through improper integrals is not explained. This seems to be the rule rather than the exception in Calculus textbooks. It is the same with Single variable calculus: Early transcendentals by Stewart (2011), the textbook used by the participants of this study.

The limit of Riemann sums in the area problem, $\lim _{n \rightarrow \infty} \sum_{i=1}^{n} f\left(x_{i}^{*}\right) \Delta x_{i}$, looks like the sum of an infinite series, and some Calculus textbooks discuss sigma notation, including infinite summation $\sum_{n=1}^{\infty} a_{n}$, just before the area problem is discussed. But in Calculus textbooks, even the ones that discuss sigma notation and $\sum_{n=1}^{\infty} a_{n}$, infinite series is discussed in a later section, after area and volume sections, including the sections that cover areas and volumes of unbounded regions, and with no apparent connection to area and volume calculations. Such connection is introduced in the integral test, which links convergence of improper integrals of certain functions over infinite intervals with 
convergence of infinite series. In the integral test the proof is done using area calculations. In this test the convergence or divergence of the improper integral of a continuous, positive and decreasing function $f$ over the interval $[N, \infty)$, where $N$ is a positive integer, is tied with the convergence or divergence of the infinite series $\sum_{n=N}^{\infty} a_{n}$ where $a_{n}=f(n)$. It can be shown that $\sum_{n=N+1}^{\infty} a_{n} \leq \int_{N}^{\infty} f(x) d x \leq \sum_{n=N}^{\infty} a_{n}$ which is valid even when the improper integral diverges. The integral test can be stated as: $\int_{N}^{\infty} f(x) d x$ is convergent if and only if $\sum_{n=N}^{\infty} a_{n}$ is convergent.

This is hardly surprising because in calculating the area by a definite integral, the underlying idea is the sum of infinite series in the guise of Riemann sums. In Gabriel's horn the surface area is associated with the divergent infinite series $\sum_{n=1}^{\infty} \frac{1}{n}$ and the volume is associated with the convergent infinite series $\sum_{n=1}^{\infty} \frac{1}{n^{2}}$. This contradicts the intuitive expectation that for a horn generated by rotating a curve around the $x$-axis has either infinite surface area and infinite volume or has finite surface area and finite volume. The following two examples are consistent with such an expectation.

Example 1. The horn generated by rotating the curve $y=\frac{1}{x^{\frac{1}{2}}}$ for $x \geq 1$ around the $x$-axis has infinite surface area and infinite volume. The volume is given by

$$
\int_{1}^{\infty} \pi\left(\frac{1}{x^{\frac{1}{2}}}\right)^{2} d x=\pi \int_{1 \frac{1}{x}}^{\infty} d x=\infty
$$

and the surface area is given by

$$
\lim _{a \rightarrow \infty} \int_{1}^{a} 2 \pi\left(\frac{1}{x^{\frac{1}{2}}}\right)\left(\sqrt{1+\left(-\frac{1}{2 x^{\frac{3}{2}}}\right)^{2}} d x \geq \lim _{a \rightarrow \infty} \int_{1}^{a} 2 \pi\left(\frac{1}{x^{\frac{1}{2}}}\right)=\lim _{a \rightarrow \infty} 2 \pi\left[2 x^{\frac{1}{2}}\right]_{1}^{a}=\lim _{a \rightarrow \infty} 2 \pi\left[2 a^{\frac{1}{2}}-1\right]=\infty .\right.
$$

Example 2. The horn generated by rotating the curve $y=\frac{1}{x^{2}}$ for $x \geq 1$ around the $x$-axis has finite surface area and finite volume. The volume is given by

$$
\int_{1}^{\infty} \pi\left(\frac{1}{x^{2}}\right)^{2} d x=\pi \int_{1}^{\infty} \frac{1}{x^{4}} d x=\frac{\pi}{3}
$$

and the surface area is given by $\lim _{a \rightarrow \infty} \int_{1}^{a} 2 \pi\left(\frac{1}{x^{2}}\right)\left(\sqrt{1+\left(-\frac{2}{x^{3}}\right)^{2}} d x \leq \lim _{a \rightarrow \infty} \int_{1}^{a} 2 \pi\right.$ $\left(\frac{\sqrt{5}}{x^{2}}\right)=2 \sqrt{5 \pi} \int_{1}^{\infty} \frac{1}{x^{2}} d x=2 \sqrt{5 \pi}$.

Computing the area or volume of an unbounded region by improper integrals is a generalization of the attributes of area and volume. These unbounded regions, like the Gabriel's horn, are actually infinite in length. Gabriel's horn, as Torricelli himself pointed out, is an extension of our idea of a solid that has finite dimensions. In generalizing area and volume concepts to these extended solids through improper integrals the notion of an infinite sum or sum of an infinite series is used indirectly and it is almost hidden. But it is apparent in the integral test. 
Historically, the notion of an infinite sum or sum of an infinite series took a long time to develop and there were many epistemological obstacles that had to be overcome. For example Grandi's series, $1-1+1-1+\ldots$, provoked controversy among the leading mathematicians in the eighteenth century. This series is divergent according to the modern definition of convergence of a series, but Leibnitz argued that since in the sequence of partial sums $1,0,1,0, \ldots$ the values 0 and 1 are equally probable and therefore their arithmetic mean $1 / 2$ is the most probable value for the sum. This was accepted by James Bernoulli, John Bernoulli, Daniel Bernoulli, Lagrange and Poisson. Even Euler argued that the sum should be $1 / 2$ but he used power series in his argument (Kline 1990; Bagni 2005).

\section{Painter's Paradox, Generalised Attributes and Infinite Series}

In summary, the Painter's Paradox is based on the fact that Gabriel's horn has infinite surface area and finite volume. The paradox emerges when we attribute finite contextual interpretations of area and volume to an intangible object of Gabriel's horn. This is what underlies the Painter's Paradox. Mathematically, this paradox is a result of generalized area and volume concepts using integral calculus, as the Gabriel's horn has a convergent series associated with volume and a divergent series associated with surface area. Linking the volume and the area of Gabriel's horn to infinite series may settle the paradox, or at least connect the perceived paradox to the counterintuitive fact that some infinite increasing series are converging, while others are diverging.

\section{The Study}

We begin this section by introducing the participants and providing a very brief summary of some of their skills acquired in the Calculus course and relevant to our study. We then introduce theoretical considerations that guided our analysis and present our research questions. The subsequent data analysis is structured according to the themes identified in the data.

\section{Participants and the Setting}

Participants in our study were 12 undergraduate students enrolled in a Calculus course. At the time of the study they were familiar with integral calculus techniques in calculating volumes and surface areas of surfaces of revolution. They were using the textbook Single variable calculus: Early transcendentals by Stewart (2011). They could set up an integral and calculate the area bounded by the curves $y=f(x), y=g(x)$, and the lines $x=a, x=b$ where $f$ and $g$ are continuous functions and $f(x) \geq g(x)$ on $[a, b]$ Also they could set up an integral and calculate the volume (by disc or cylindrical shell methods) or surface area of the solid generated when this region is rotated around the $x$ axis. They also could calculate volume or surface area of unbound regions, like Gabriel's horn.

The participants were presented the Painter's Paradox with detailed mathematical justifications of computing the volume and surface area of the Gabriel's 
horn and showing that its volume is finite while the surface area is infinite. The volume calculation using only the disc method was given, as this method seemed to be simpler than the cylindrical shell method. The participants were asked to respond in writing explaining the perceived paradox. The Task is presented in Fig. 2.

Shortly after completing the Task the participants were interviewed by the first author. The interviews aimed at probing the participants' written responses and seeking

\section{Gabriel's Horn Task}

25. If the region $\mathscr{R}=\{(x, y) \mid x \geqslant 1,0 \leqslant y \leqslant 1 / x\}$ is rotated about the $x$-axis, the volume of the resulting solid is finite (see Exercise 63 in Section 7.8). Show that the surface area is infinite. (The surface is shown in the figure and is known as Gabriel's horn.)

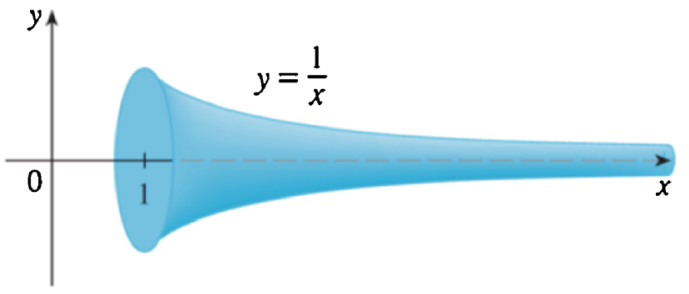

The volume of the solid is given by

$\lim _{a \rightarrow \infty} \int_{1}^{a} \pi\left(\frac{1}{x}\right)^{2} d x=\lim _{a \rightarrow \infty}\left(\pi \int_{1}^{a} \frac{1}{x^{2}} d x\right)=\lim _{a \rightarrow \infty}\left(\pi\left[-\frac{1}{x}\right]_{1}^{a}\right)=\lim _{a \rightarrow \infty}\left(\pi\left[1-\frac{1}{a}\right]\right)=\pi$

And the surface area is given by $\lim _{a \rightarrow \infty} \int_{1}^{a} 2 \pi\left(\frac{1}{x}\right)\left(\sqrt{1+\left(-\frac{1}{x^{2}}\right)^{2}} d x\right.$.

But $\lim _{a \rightarrow \infty} \int_{1}^{a} 2 \pi\left(\frac{1}{x}\right)\left(\sqrt{1+\left(-\frac{1}{x^{2}}\right)^{2}} d x>\lim _{a \rightarrow \infty} \int_{1}^{a} 2 \pi\left(\frac{1}{x}\right) d x=\lim _{a \rightarrow \infty} 2 \pi[\ln x]_{1}^{a}=\lim _{a \rightarrow \infty} 2 \pi[\ln a-\ln 1]=\infty\right.$.

So the volume of the Gabriel's horn is finite but its surface area is infinite. So to paint the inner surface of the horn we need an infinite amount of paint. But we could pour $\pi$ amount of paint into the horn and then empty the horn so that the inner side is painted. What do you think of the paradoxical situation here? Please write down your thoughts. Please note that the above calculations of volume and surface area of the horn are correct.

Fig. 2 Gabriel's Horn Task 
additional articulation of their explanations. The interviews were audio recorded and transcribed.

\section{Theoretical Considerations}

We rely on several theoretical frameworks in our data analysis - epistemological obstacles by Brousseau (1983), platonic and contextual distinction by Chernoff (2011), and reducing abstraction by Hazzan (1999).

\section{Epistemological Obstacles}

Brousseau's theoretical construct of epistemological obstacles is based on the assumption that knowledge is an optimal solution in a system of constraints. In his view, knowledge is a solution to a problem independent of the solver. He characterized epistemological obstacles as "those [obstacles] that cannot and should not be avoided, precisely because of their constitutive role in the knowledge aimed at. One can recognize them in the history of the concepts themselves" (Brousseau, as quoted in Radford et al. 2000, p. 163). Brousseau (1983) classified sources behind students' recurrent and non-aleatorical mistakes in learning mathematics as follows:

(1) an ontogenetic source (related to the students' own cognitive capacities, according to their development);

(2) a didactic source (related to the teaching choices);

(3) an epistemological source (related to the knowledge itself).

So, epistemological obstacles arise from the third source. Brousseau suggests that they can be detected through a confrontation of the history of mathematics and today's students' learning mistakes. Combining history and psychogenesis Sfard (1995) noted:

Indeed, there are good reasons to expect that, when scrutinized, the phylogeny and ontogeny of mathematics will reveal more than marginal similarities. At least, this is what follows from the constructivist view according to which learning consists in the reconstruction of knowledge. (p. 15)

We consider the area-volume relationship of the Gabriel's horn as an epistemological obstacle, as it created a considerable debate among mathematicians at the time.

\section{Contextualization}

We also rely on the theoretical constructs introduced by Chernoff (2011) in distinguishing between platonic and contextualized situations or objects. Chernoff distinguished between platonic and contextualized sequences in the relative likelihood tasks in probability. A platonic sequence is characterized by its idealism. "For example, a sequence of coin flips derived from an ideal experiment (where an infinitely thin coin, which has the same probability of success as failure, is tossed repeatedly in perfect, independent, identical trials) would represent a platonic sequence" (p. 4). But, a contextualized sequence is characterized by its 
pragmatism. For example, "the sequence of six numbers obtained when buying a (North American) lottery ticket (e.g., 4, 8, 15, 16, 23, 42)" (p. 4) would represent a contextualized sequence.

Gabriel's horn is a platonic object. It is formed by rotating a breathless and infinitely long curve. But Painter's Paradox is presented in a 'realistic' context and its resolution requires de-contextualization from the physical reality, which is assuming that the paint can reach every part of the Gabriel's horn and time is not a factor.

\section{Reducing Abstraction}

Hazzan (1999) introduced a framework of reducing abstraction, initially considering students' work in an abstract algebra course. The main tenet of this framework is that when individuals engage in novel problem solving situations, their attempts to make sense of unfamiliar and abstract concepts can be described through different means of reducing the level of abstraction of those concepts. That is, reducing the level of abstraction is a mental activity of coping with abstraction. In an attempt to deal with a situation, students may approach a task on the lower level of abstraction than the level expected by the teacher or the task itself. Hazzan's descriptive framework utilizes different concepts of abstraction and elaborates on three ways:

1) Abstraction level as the quality of the relationships between the object of thought and the thinking person.

2) Abstraction level as reflection of the process-object duality

3) Abstraction level as the degree of complexity of the mathematical concept.

Here we focus mainly on interpretation (1), which acknowledges that different people may approach mathematical objects on different level of abstraction. This is consistent with Hershkowitz et al. (2001) perspective that emphasises the learner's role in the abstraction processes. They claim that "abstraction depends on the personal history of the solver" (p. 197). The tendency to interpret unfamiliar within familiar terms and experiences, which are more concrete to an individual, is consistent with this interpretation. Further, Mamolo and Zazkis (2014) considered contextualization as a possible way of reducing the level of abstraction by shifting from an unfamiliar (or less familiar) to a familiar situation.

As mentioned above, the paradox is presented in a 'realistic' context, but decontextualization of a paradox from the physical reality is essential for its resolution. However, in analysing the paradox students may attend to it in different levels of abstraction. Relying on a familiar context and bringing into consideration contextual elements reduces the level of abstraction in considering the task.

\section{Research Questions}

Using the theoretical considerations presented above, our study looks at students' attempts at resolving the Painter's Paradox. Painter's Paradox is different from other paradoxes of infinity used in mathematics education research to explore conceptions of infinity as it does not involve infinite subdivision of space or time. This paradox involves a solid, which is not bounded from every side and has finite volume. Our 
Table 1 Themes in students' responses

\begin{tabular}{lll}
\hline Theme & Subthemes & Number of responses \\
\hline Epistemological obstacles & & 6 \\
Inadequacy of mathematics & Calculus will be developed further & 1 \\
& Paradoxes are part of mathematics & 2 \\
Contextualization & Horn cannot be filled in a finite time & 3 \\
& Paint will get stuck & 4 \\
& Cannot paint as the horn cannot be seen & 1 \\
\hline
\end{tabular}

study explores the specific challenges faced by students in resolving the Painter's Paradox. We address the following interrelated research questions:

- How do undergraduate calculus students attempt to resolve the Painter's Paradox? In particular, how do they deal with a solid, which is infinite in length/surface area but has a finite volume?

- What challenges do they face? What mathematical and what contextual considerations do students rely on in dealing with the challenges?

\section{Data Analysis}

We examined all students' written responses to the task as well as their further explanations, elaborations or clarifications in the interviews. Several interwoven themes were identified; their frequency of occurrence is presented in Table 1. Note that more than one theme was present in some of the responses.

We now present analysis of the data according to the above themes.

\section{Epistemological Obstacles}

In identifying the theme of epistemological obstacles, we looked for any firmly held notions that indicate that the infinitely long Gabriel's horn should have infinite volume. Half of the students had trouble dealing with Gabriel's horn having a finite volume. Like the seventeenth century mathematicians and philosophers they reacted in disbelief. For example, Sean ${ }^{1}$ wrote:

if it is infinitely long it's going to have infinite volume. So I don't know how we are getting a finite volume.

Bruce acknowledged the counterintuitive nature of the result:

It goes against something that really is intuitive. Like in our minds we all know that if something has a finite volume usually it has a finite surface area.

\footnotetext{
${ }^{1}$ All names are pseudonyms
} 
This tendency to extrapolate from real life experiences of finiteness to an unbounded Gabriel's horn seemed overwhelming in spite of their experience and familiarity with convergent improper integrals. For example, one of the results the students were familiar with is that $\int_{1}^{\infty} \frac{1}{x^{p}} d x$ is convergent for $p>1$, where $\int_{1}^{\infty} \frac{1}{x^{p}} d x$ is the area under the curve $y=\frac{1}{x^{p}}$ for $x \geq 1$. However, participants did not rely on this knowledge in discussing the paradox.

Kevin's resistance to accepting Gabriel's horn having finite volume is captured in the following interview excerpt:

Interviewer: What is paradoxical about this horn?

Kevin: $\quad$ Paradox is about the that integral $1 / \mathrm{x}$ ur geometrical shape of $1 / \mathrm{x}$ ur at infinity ah infinite area either, either cross sectional area or surface area of revolution but finite volume.

Interviewer: Why is it paradoxical?

Kevin: $\quad$ Because we can't imagine this geometrical figure it does not make sense to us, when I try to integrate this $1 / \mathrm{x}$ up to very large numbers I get, I got larger, larger area cross sectional area of the shape but I always get this finite volume so.., and humans cannot apprehend this idea.

Interviewer: Can you tell me more why humans cannot apprehend this?

Kevin: $\quad$ Because ..., we have I personally have this idea of relation between cross sectional area of a shape and its volume and in my understanding if I add some more and more area I should also add some volume.., and I cannot imagine this situation where I keep adding this area and this area gets very large but my volume stays the same.

Kevin seems to think that if you keep adding to something it has to get very large. He also has difficulty in seeing that a positive increasing infinite series can converge. These students' reactions are in fact predictable, especially when we turn to Sfard's (1995) observation:

[...] natural resistance to upheavals in tacit epistemological and ontological assumptions, which so often obstructed the historical growth of mathematics, can hardly be prevented from appearing in the classroom. (p.17)

We recognize in the ideas presented by Sean and Kevin a reducing of abstraction level by referring to a familiar and less complex situation. They refer to their experience with volume and area of finite objects, which present less complexity than infinitely long intangible object of Gabriel's horn. 


\section{Inadequacy of Mathematics}

Rather surprisingly, three participants treated this paradoxical situation as a consequence of the inadequacy of mathematics. Kevin expressed a belief that in a "more complicated" Calculus we will not have this paradox:

I feel like this part of Calculus is not perfected yet. I mean, people always tend to think that their contemporary science is very good, but then new generations of scientists prove them wrong. You know that math is just a language created by humans to describe certain aspects of life. And human brain is not perfect, so there is no reason why math must work perfectly in every possible situation. I believe that in the future someone can come up with a new more complicated Calculus, that will not have such paradoxes in it. Maybe it won't even be based on numbers like our math is.

Unlike Kevin, Bryan is not seeking a different Calculus, but rather refers to a similar situation within mathematics. Bryan's response seems to suggest that the paradox is in the mathematics itself, as a similar paradoxical situation exists in infinite series. Bryan wrote that "the paradoxical situation is correct as defined by the solutions" and that it reminded him of the paradoxical situation that arises in the summation of an infinite geometric series:

a geometrically infinite series never ends in the amount of terms in the series, however, the sum of all these terms is a finite number.

His ideas resonate with Wallis's, who shared Leibniz' opinion, as mentioned earlier, that Torricelli's solid having a finite volume was as surprising as the infinite series $\frac{1}{2}+\frac{1}{4}+\frac{1}{8}+\frac{1}{16}+\frac{1}{32}+\ldots$ being equal to 1 .

As stated above, one way of reducing abstraction is by changing the quality of the relationships between the object of thought and the thinking person through connecting the unfamiliar to the familiar. Here Kevin refers to the familiar notion that mathematics is an evolving and growing body of knowledge and, implicitly acknowledging that some problems in mathematics waited for centuries for their solutions, he leaves the resolution of the paradox to scientists of future generations. However, for Bryan, a familiar connection refers back to previously experienced seemingly paradoxical situations.

\section{Contextualization}

The paradox itself is presented in a contextual setting of painting the horn. However, several responses indicate further contextualization of the paradox, that is, adding contextual considerations that are not present in the description of the paradox.

Three students suggested that the horn cannot be filled in a finite time. David wrote and further reiterated that "you can never fill the horn with paint since the horn is $\infty$ long. Time must be a factor". The time factor is an additional contextualization to demonstrate that the set task is impossible to carry out in reality. 
Alysa suggested that the horn cannot be painted "because paint molecules will get stuck in the horn". And while Alysa referred to molecules in her explanation, Peter mentioned "atomic level". For Peter, the horn cannot be painted because at the atomic level you cannot see the surface:

that surface.., when it goes to that atomic level..., the size of that atom of Hydrogen that surface is going to be invisible.., you can't see it anymore.., we can't see it.., how can you paint something you can't see? I can only paint something that we are used to seeing color yellow blue so they have to have some kind of size when it goes smaller than the size of some microscopic.., we can't see any more so we can't see it.., we can't paint it!

Peter's response shows a very strong form of contextualization - atoms are invisible, one cannot paint what he can't see - and highlights the difficulty in decontextualizing the Painter's Paradox.

Bryan attempted to explain paradoxical finite volume - infinite surface area situation by connecting it to tangible and familiar Silly Putty he used to play with. "What I most often do is roll the Silly Putty into a long cylindrical roll of Silly Putty and continue to do so until I had a very thin roll of Silly Putty" he wrote. He explained how a finite volume can have an infinite surface area: "using a finite amount of Silly Putty, we could theoretically roll the silly putty to an infinitely thin thickness, and length [infinite surface area] [...] Therefore, a finite amount of material can have an infinite surface area."

By referring to a familiar toy with unusual physical properties Bryan seemed to have unraveled the paradox. However, in the interview conducted immediately after the written task he doubted the idea that the horn could be painted with a finite amount of paint. He acknowledged that the mathematics is correct. But he added that it does not make sense: "that is impossible! [...] if you were to paint this room and it is growing infinitely large, you know you have only 3.14 l of paint, it does not make sense". The bold headed "that is impossible" was claimed in disbelief. Bryan seemed to be in an intellectually unbearable situation, or in a cognitive conflict, in spite of his earlier observation that a finite amount of material can have an infinite surface area.

We note that by adding unintended contextual features students reduce the level of abstraction in an attempt to explain the paradox: Students consider painting a horn not as a "thought experiment" that relates to an imaginary platonic object (unfamiliar) but as a realistic task to be competed in a finite time interval using a real paint (familiar). They refer to familiar experience (Silly Putty) and interpret the resulting calculation with familiar measurements $(3.14$ 1). Furthermore, in their attempts to deal with infinitely small quantities they refer to familiar, or at least previously explored, concepts, such as atoms and molecules.

\section{A Possible Resolution?}

While comparisons to "familiar and very small" concepts, such as atoms and molecules" led Alysa and Peter to reject the idea that the horn can be painted, Bruce's consideration of "infinitely small" hints at a possible resolution, which we consider in this section. 
After noting that Gabriel's horn having a finite volume and infinite surface area is counterintuitive (this was noted above as epistemological obstacle), Bruce added: "If the math is right which we are told that it is then ... because often times arrive at a paradox so from my experience like dealing with stuff from Physics I know ... I am not too foreign to the idea of something being one thing and at the same time another thing." In this claim Bruce acknowledges a possibility of a paradox in mathematics and a connection to a familiar situation in Physics related to a change in states of matter.

At the end of his lengthy written response Bruce wrote about the connection between area and volume, and Riemann sums. He noted that the area and volume are defined using Riemann sums. To say that the volume is finite, he wrote, is to say that the [Riemann] sum converges. And to say that the surface area is infinite is to say that the [Riemann] sum diverges. His thinking is captured well in the following excerpt:

I think the reason we define 3D volume and surface area this way is because it makes intuitive sense. But not all Riemann sums are well behaved - some diverge and our intuition stops us from accepting so called paradoxes, like Gabriel's Horn, as reality in the world of abstract mathematics.

Bruce's written response is in accord with mathematical decontextualized resolution of the Painter's Paradox presented above. During the interview, when asked to elaborate on his ideas, Bruce took a different approach. He suggested that there could be two kinds of paint, one that occupies volume and one that covers surface area. This was his apparent attempt to achieve an equilibrium focusing on the dimensional difference between the surface area and volume.

Bruce then reduced the abstraction of the paradoxical finite-volume-infinite-surface area situation by connecting it to something tangible:

some finite things can occupy infinite surface area, like let's say I have a cube let's say I squash with some kind of really strong plate, so that no matter how thick it is it can always become thinner then the surface area... Yeah, it would be finite volume but it would spread over an infinite surface area if you think of painting that way $[. .$.$] what is thickness of an area it would have to be infinitely thin...$

We note that the level of abstraction in his description is reduced by referring to the process of making something "thinner" and noting that "it can always become thinner", rather than to a related "infinitely thin" object. Bruce seems to have made a connection between his observation "some finite things can occupy infinite surface area" and painting the infinite surface area of the horn with a finite amount of paint. The above segment in the transcript happened at the very end of the half an hour interview and the bold headed yeah was emphatic. This seems to be an indication of a new realization. Bruce used the words infinitely thin repeatedly in explaining how a finite volume can have infinite surface area, and this seems to show the emergence of conceptions of infinitely small in his thinking.

Bruce's written response connects the resolution of the paradox to the infinite series on which the calculation of the integral is based. However, his new realization and his reference to "infinitely thin paint" is in accord with an additional resolution, suggested 
in Gethner (2005). Gethner (2005) explains that the Painter's Paradox is based on the "realistic" consideration that the thickness of paint is the same on the whole painted area, as is reasonable to assume in painting a wall. The alternative resolution does not reject contextualization, but includes an imaginary context, a "mathematical" paint of infinitely thin layers. The inner surface of the Gabriel's horn can be painted with $\pi$ amount of paint (or less) if the thickness of the paint layer at $x$ is smaller than or equal to $\frac{1}{x}$. With this assumption the paint, when poured into the Gabriel's horn, can reach every point of the infinite surface area. So the amount (volume) of paint needed in this case, that is, if the thickness of the paint at layer $x$ is less than or equal to $\frac{1}{x}$, is less than or equal to the volume of the horn, which is $\pi$.

\section{Discussion and Conclusion}

Painters Paradox is an example of a seemingly paradoxical situation that involves infinity and can be resolved with the help of Calculus. In what follows we consider this paradox within other paradoxes that have similar attributes. We then turn the discussion on to the particular results of this study, focusing on the cognitive conflict presented by the paradox and participants' reactions to the paradox in their attempt to resolve the cognitive conflict. We conclude with some implications for teaching Calculus. We suggest a possible emphasis in Calculus instruction that could deepen students' understanding of frequently used approaches to determine surface area and volume of objects resulting from rotating graphs of functions.

\section{On Paradoxes and Context}

Though there is no rigorous conventional definition for the term paradox, its use in mathematics may refer to a sound argument that leads to a contradiction, also known as antinomy, or a statement that is counterintuitive or seemingly unacceptable. Among various kinds of known paradoxes, we note a distinction between logical fallacies and counterintuitive, inconsistent, puzzling or seemingly absurd situations, in which there is no apparent mathematical contradiction. ${ }^{2}$ The well-known Liar's Paradox (e.g., "I'm lying"), or self-referential statements (e.g., "The least natural number not definable in fewer than thirteen English words.") and their variations are examples of the former. The Painter Paradox, as well as most paradoxes involving infinity, is an example of the latter. In this kind of paradoxes a seemingly paradoxical situation is connected to the counterintuitive aspects of infinity, especially when the "story" of the paradox in embedded in a semi-realistic context. Consider for example the story of Achilles and the tortoise paradox. While the situation of Achilles being unable to reach the tortoise is counterintuitive, the conventional resolution abstracts the story of the "race" and considers the converging geometric series. This points to the general heuristics: resolution of some of the paradoxes involves decontextualization, that is, consideration of the "givens" outside of the story line. This is indeed the case in the conventional resolution of the Painters' Paradox.

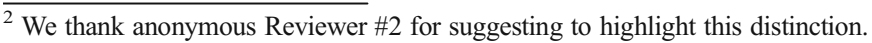


Painter's Paradox highlights the counterintuitive aspect of the Gabriel's horn, or the Torricelli's infinitely long solid, having a finite volume and infinite surface area. Of note is that the Gabriel's horn is not the only mathematical object, which is both finite and infinite when different dimensions are considered. Koch's snowflake, based on Koch's curve, is another example of a mathematical object that has both finite and infinite attributes in different dimensions. To elaborate, the area of this fractal is calculated by a convergent series and its perimeter is given by a divergent series. So Koch's snowflake has a perimeter of infinite length that encloses a finite area. Of interest here is that the finite attribute (area) is of a higher dimension than the infinite attribute (length or perimeter). This is similar to the case of Gabriel's horn, where the finite attribute (volume) is of a higher dimension than the infinite attribute (surface area).

\section{Cognitive Conflict and Different Ways to Deal with Tension}

Painter's Paradox, as well as any other mathematical paradox, presents a cognitive conflict to a learner. Movshovitz-Hadar and Hadass (1991) indicate that "as long as a person cannot resolve a paradox, he or she is in a state of cognitive conflict" (p. 80). Cognitive conflict, according to Piaget, is a state of disequilibrium between assimilation and adaptation-accommodation. This state is ordinarily inconvenient and creates tension. All of the participants seemed to be experiencing a cognitive conflict dealing with the Painter's Paradox. Students faced the seemingly paradoxical claims that an infinitely long solid has a finite volume and an infinite surface area, and the counterintuitive nature of this realization was reinforced by the story of paint. In students' thinking cognitive conflicts apparently emerged between competing real life intuitions, and logical and mathematical entailments of the paradox.

Movshovitz-Hadar and Hadass (1991) point out that the state of cognitive conflict and the tension it creates stimulates an attempt to get out of it and achieve a new equilibrium with a more advanced mental structure. While all students attempted to achieve equilibrium, their chosen approaches to deal with the situation differed.

Participants, while experiencing cognitive conflict in trying to resolve the paradox, appeared to ease the resulting tensions by bringing in a variety of considerations. As shown above, some participants referred to the impossibility of the presented situation and in such echoed epistemological obstacles experiences by mathematicians of the 17th century. Others acknowledged the paradox, but accepted it as a part of not yet fully developed mathematics (see Kevin above) or connected the paradox to other seemingly paradoxical situations. For example, Bryan made an important connection when he said that the paradoxical situation in the Gabriel's horn reminded him of the paradoxical situation that arises in the summation of an infinite geometric series. However, this connection to convergent series was not fully developed as it served as an additional example of counterintuitive nature of mathematics, rather than a phenomenon that explains and resolves the paradox.

A majority of participants added contextual considerations, suggesting that it was impossible to paint the horn due to time limits, thickness of paint, or the painter's ability to see the object. These attempts to bring in realistic considerations point to students' tendency to attack the task on a reduced level of abstraction, without explicit attention to the fact that the presented object does not exist in the physical reality. So rather than 
resolving the paradox mathematically, these participants rejected the viability of the presented "story".

Bruce appeared to be the only participant who generated ideas in accord with decontextualized resolution that attends to convergent and divergent series, as well as with the alternative resolution that introduced imaginary-magic context of infinitely thin paint applied at not uniform paint layers (thickness of the paint layer at $x$ is less than or equal to $\frac{1}{x}$ ).

Decontextualization of Painter's Paradox seemed to be difficult as the majority of participants found various ways of contextualizing the paradox further and in such avoiding its resolution. But the ability to consider something platonically is an important skill to acquire in mathematics. In fact, Mamolo and Zazkis (2008) in their study of several paradoxes of infinity, argued for an instructional approach that helps students separate their realistic and intuitive considerations from conventional mathematical ones.

\section{Contextualization and Abstraction}

In applying a theoretical lens of reducing abstraction, we noted that contextualization is one possible avenue of reducing abstraction. When abstraction is considered as a quality of relationship between the object of thought and the thinking person, a context helps in the shift towards a familiar, or a more familiar, situation. However, a conventional resolution of the paradox involves abstracting the context and considering the situation stripped from the contextual story of a painter. The tension between contextualization and abstraction can serve as a platform for further exploration, as well as for an extended pedagogical attention.

In addition to the context of the story of paining the horn, there are contextual elements of area and volume that influenced the participants' reactions. That is to say, while the participants attempted to elaborate and contextualize further the story of a painter, the elements of area and volume were "taken as shared". That is to say, some shared understanding of these attributes was assumed as not requiring any explanation or elaboration. This could have been yet another implicit contextualization and reducing the level of abstraction by considering area and volume of familiar finite objects, rather than attributes as related to an abstract imaginary object of the horn. We note that understanding of generalized attributes of volume and area could be the key for resolving the paradoxical situation of the Painter's Paradox. However, attention to this subtlety requires a considerable attention in approaching definite integrals in the study of Calculus. We attend to this issue in the next section.

\section{Obstacle, not Only Epistemological}

Associating a finite attribute with something infinite, as in the case of Gabriel's horn, is clearly an epistemological obstacle as the difficulty of our participants replicates the development of the mathematical knowledge through history. But this also can be seen as a didactic obstacle, as Calculus textbooks - and consequently many instructors - do not emphasize that calculating areas and volumes of unbounded regions through improper integrals generalizes the concepts of area and volume to unbounded regions. Bråting (2012) noted that one problem of this lack of explanation could be 
that students might think that it is possible to understand that Gabriel's horn has finite volume based on intuitions developed by dealing with everyday objects.

The observation made by Bryan - that the infinitely long Gabriel's horn having a finite volume is as paradoxical as a geometric series with infinitely many terms adding up to a finite number - is an excellent starting point in drawing an analogy between the two situations. However, the volume of the Gabriel's horn is not given by the sum of a decreasing geometric sequence, but by the convergent series $\sum_{n=1}^{\infty} \frac{1}{n^{2}}$. The connection to converging series is a key to understanding why Gabriel's' horn has a finite volume.

A useful didactic approach in Calculus teaching would be to reinforce the connection between definite integrals and Riemann sums, so that Calculus students would understand and remember why definite integral gives area and volume, and not just apply the calculation as a rule without reason. What is badly missing in Calculus textbooks is the explicit attention to the idea that using integrals in calculations of area and volume of unbounded regions is a generalization of these attributes, area and volume, to unbounded regions. Didactic attention to the connection between such generalizations and infinite series is essential for students to be able to strive for and derive paradox resolution, rather than accept paradoxes as inadequacies of mathematics.

Of course, the viability of the suggested approach is a subject for future investigation. Painter's Paradox, as well as other paradoxes of infinity, will keep offering puzzling engagement, regardless of one's ability to derive a conventional resolution. However, the ability to resolve the paradox by rejecting the context and applying concepts of Calculus does not diminish its attractive nature. We believe that the experienced conflict and the achieved equilibrium may add to the appreciation of mathematical beauty.

\section{References}

Adams, R. A. (2002). Calculus - a complete course. Addison Wesley.

Adams, R. A., \& Essex, C. (2010). Calculus: A complete course. Reading: Pearson Addison-Wesley.

Bagni, G. T. (2005). Infinite series from history to mathematics education. International Journal for Mathematics Teaching and Learning, 1473-0111.

Bråting, K. (2012). Visualizations and intuitive reasoning in mathematics. The Montana Mathematics Enthusiast, 9(1\&2), 1-18.

Brousseau, G. (1983). Les obstacles épistémologiques et les problèmes en mathématiques. Recherches en Didactique des Mathématiques, 4(2), 165-198.

Carroll, M. T., Dougherty, S. T., \& Perkins, D. (2013). Indivisibles, infinitesimals and a tale of seventeenthcentury mathematics. Mathematics Magazine, 86(4), 239-254.

Chernoff, E. (2011). Investigating relative likelihood comparisons of multinomial contextual sequences. Proceedings of CERME 7.

Dubinsky, E., Weller, K., McDonald, M. A., \& Brown, A. (2005). Some historical issues and paradoxes regarding the concept of infinity: an APOS-based analysis: part 1. Educational Studies in Mathematics, $58,335-359$.

Fleron, J. F. (1999). Gabriel's wedding cake. The College Mathematics Journal, 30, 35-38.

Gethner, R. M. (2005). Can you paint a can of paint? The College Mathematics Journal, 36(5), 400-402.

Hazzan, O. (1999). Reducing abstraction level when learning abstract algebra concepts. Educational Studies in Mathematics, 40(1), 71-90.

Hershkowitz, R., Schwarz, B. B., \& Dreyfus, T. (2001). Abstraction in context: epistemic actions. Journal for Research in Mathematics Education, 32, 195-222. 
Kim, D. J., Ferrini-Mundy, J., \& Sfard, A. (2012). How does language impact the learning of mathematics? Comparison of English and Korean speaking university students' discourses on infinity. International Journal of Educational Research, 51, 86-108.

Kleiner, I., \& Movshovitz-Hadar, N. (1994). The role of paradoxes in the evolution of mathematics. The American mathematical monthly, 101(10), 963-974.

Kline, M. (1990). Mathematical thought from ancient to modern times (Vol. 3). New York: Oxford University Press.

Luis, E., Moreno, A., \& Waldegg, G. (1991). The conceptual evolution of actual mathematical infinity. Educational Studies in Mathematics, 22(3), 211-231.

Mamolo, A., \& Zazkis, R. (2008). Paradoxes as a window to infinity. Journal for Research in Mathematics Education, 10(2), 167-182.

Mamolo, A., \& Zazkis, R. (2014). Contextual considerations in probabilistic situations: An aid or a hindrance?. In Probabilistic Thinking (pp. 641-656). Springer Netherlands.

Mancosu, P., \& Vailati, E. (1991). Torricelli's infinitely long solid and its philosophical reception in the seventeenth century. Isis, 82(1), 50-70.

Movshovitz-Hadar, N., \& Hadass, R. (1990). Preservice education of math teachers using paradoxes. Educational Studies in Mathematics, 21(265), 87.

Movshovitz-Hadar, N., \& Hadass, R. (1991). More about mathematical paradoxes in preservice teacher education. Teaching and Teacher Education, 7(I), 79-92.

Netz, R., \& Noel, W. (2011). The Archimedes codex. Hachette UK.

Núñez, R. (1994). Cognitive development and infinity in the small: paradoxes and consensus. In Proceedings of the Sixteenth Annual Conference of the Cognitive Science Society (pp. 670-674). Hillsdale, NJ: Lawrence Erlbaum Associates.

Quine, W. V. O. (1976). The ways of paradox: and other essays. Harvard University Press. Zeno's paradox of plurality and proof by contradiction (2001). Mathematical Connections. Series II(1), 3-16.

Radford, L., Boero, P., \& Vasco, C. (2000). Epistemological assumptions framing interpretations of students understanding of mathematics. In J. Fauvel, J. A. Maanen, \& J. A. van Maanen (Eds.), History in mathematics education: An ICMI study (pp. 162-167). Dordrecht: Kluwer Academic Publishers.

Sfard, A. (1995). The development of algebra: confronting historical and psychological perspectives. The Journal of Mathematical Behavior, 14(1), 15-39.

Stewart, J. (2011). Single variable calculus: Early transcendentals. Pacific Grove: Brooks/Cole Publishing Company. 